\title{
Circle of Willis Morphology in Primary Intracerebral Hemorrhage
}

\author{
Mangmang $\mathrm{Xu}^{1} \cdot \mathrm{Qian}_{\mathrm{Wu}}{ }^{1}$ - Yajun $\mathrm{Cheng}^{1}$ - Shuting Zhang ${ }^{1} \cdot$ Wendan Tao $^{1}$. Shihong Zhang ${ }^{1} \cdot$ Deren Wang ${ }^{1}$. \\ Ming Liu ${ }^{1} \cdot \mathrm{Bo} \mathrm{Wu}^{1} \mathbb{0}$
}

Received: 12 December 2021 / Revised: 21 January 2022 / Accepted: 9 February 2022 / Published online: 19 February 2022 (c) The Author(s) 2022

\begin{abstract}
We aimed to study the distribution of Circle of Willis (CoW) morphology and its association with intracerebral hemorrhage (ICH) etiology and cerebral small vessel disease (CSVD) burden. Patients with primary ICH who had brain MRIs were consecutively enrolled between March 2012 and January 2021. CoW morphology, CSVD features and the combined CSVD burden (including global CSVD burden, total hypertensive arteriopathy [HA] burden, and total cerebral amyloid angiopathy [CAA] burden) were assessed. CoW morphology included poor CoW (defined as CoW score 0-2), incomplete CoW, and complete fetal-variant of the posterior communicating artery (CFPcoA). Among 296 patients enrolled, 215 were included in the analysis. There was no significant difference among HA-, CAA-, and mixed-ICH in each CoW morphology. Exploratory subgroup analyses suggested that poor $\mathrm{CoW}$ was associated with a greater incidence of HA-ICH and low incidence of mixed ICH in patients aged $<60$ years, while mixed ICH occurred more frequently in patients with CFPcoA, especially in those without hypertension history (all $p<0.050$ ). Additionally, incomplete CoW was correlated with a larger incidence of lacunes (adjusted $O R$ [adOR] 2.114, 95\% CI 1.062-4.207), microbleeds $\geq 5$ (adOR 2.437, 95\% CI 1.187-5.002), and therefore the combined CSVD burden (adOR 1.194, 95\% CI 1.004-1.419 for global CSVD burden, adOR 1.343, 95\% CI 1.056-1.707 for total CAA burden), independent of modifiable vascular risk factors, but not age and sex. The CoW might therefore have a potential impact on ICH etiology and is associated with a greater CSVD burden. Our findings are novel, and need to be verified in future studies.
\end{abstract}

Keywords Circle of Willis · Primary intracerebral hemorrhage · Cerebral small vessel disease $\cdot$ ICH etiology $\cdot$ Cerebral microbleeds

\section{Introduction}

Hypertensive arteriopathy (HA) and cerebral amyloid angiopathy (CAA) are two common forms of cerebral small vessel diseases (CSVD), which are responsible for the major causes of primary intracerebral hemorrhage (ICH) [1, 2]. Despite the devastating consequences of primary $\mathrm{ICH}$, the mechanisms underlying ICH are poorly understood. It is well established that hypertension is the most crucial risk factor for HA-ICH [3, 4], and an increasing burden of

Bo Wu

dr.bowu@hotmail.com

1 Center of Cerebrovascular Diseases, Department of Neurology, West China Hospital, Sichuan University, Chengdu, Sichuan Province, China
CSVD features are associated with ICH occurrence [5] and recurrence [6,7]. The distribution of CSVD features, such as lacunes, white matter hyperintensities (WMH) of presumed vascular origin, cerebral microbleeds (CMBs), cortical superficial siderosis (cSS), and enlarged perivascular spaces (EPVS) [3], is distinctive in severe HA and CAA [8], making them useful for identifying the pathological mechanisms of ICH.

The Circle of Willis (CoW) varies among the general population, and it has been hypothesized that cerebral autoregulation could be affected by the absence of communicating arteries of CoW [9]. A previous study demonstrated that an incomplete posterior $\mathrm{CoW}$ might be a factor in triggering hypertension [10]. Additionally, CoW morphology plays a fundamental role in CSVD features. In healthy individuals, incompleteness of the anterior CoW correlates with a higher frequency of deep lacunes [11]. A greater WMH burden is found in those with an incomplete CoW [9]. 
Fetal configuration of posterior $\mathrm{CoW}$ is associated with a decreasing load of small- and medium-size of deep WMH (DWMH), but not large WMH in patients with clinical manifestations of atherosclerotic disease [12].

Because of the close relationship between CoW morphology, hypertension, and etiology-associated CSVD features, where deep lacunes are more frequent in HA-ICH [13], and the prevalence of multiple subcortical spots of WMH (DWMH) is higher in CAA-ICH [14]; there might be a potential relationship between CoW morphology and ICH etiology. In our present study, we addressed the issue of whether CoW morphology is associated with ICH etiology by comparing the CoW morphology among HA-, CAA-, and mixed-ICH, and whether it is associated with CSVD burden, individually and incorporated in the combined CSVD burden.

\section{Methods}

\section{Patient Recruitment}

We performed a retrospective analysis based on our ongoing longitudinal database collected at the West China Hospital of Sichuan University. The present study subjects were consecutive patients with a diagnosis of primary ICH who had a brain MRI to assess CSVD burden admitted to our center between March 2012 and January 2021. Patients with intracerebral hemorrhage because of hemorrhagic transformation of an ischemic infarct, brain tumor, rupture of a structural vascular lesion such as aneurysm or arteriovenous malformation, or systemic diseases such as liver cirrhosis and renal failure were not enrolled. In the final analysis, we excluded those who had history of ischemic stroke, were diagnosed as primary intraventricular hemorrhage, aged $<18$ years at stroke onset, or did not have brain CTA or MRA for CoW assessment. This study was approved by the Ethics Committee on Biomedical Research, West China Hospital of Sichuan University (number of approval document: 362th of Year 2019). The ethics committee waived the need for patient consent for the present study because of the retrospective chart review.

\section{Definitions of CoW Morphology}

Two experienced neurologists (Mangmang Xu and Qian $\mathrm{Wu}$ ) masked to clinical characteristics and ICH etiology reviewed brain CTA or MRA to assess the CoW independently and the disagreements were resolved through discussion. The median time from onset to MRA/CTA was $1.8(0.5-8.9)$ days. The presence, diameter, and length of all component vessels of the CoW were evaluated on the source images of 3D time-of-flight and maximum intensity projection images of MRA [9, 15] or source data of CTA [16]. Any vessel that was at least $0.8 \mathrm{~mm}$ in diameter was considered as present [15]. In our present study, an incomplete CoW was considered when any vessel of the CoW was absent or less than $0.8 \mathrm{~mm}$ in diameter in line with reference standards [15]. We used CoW score according to the score proposed by Kim et al. [17] to evaluate the extent of collateral circulation. $\mathrm{CoW}$ score was an ordinal scale ranging from 0 to 6 , with a formula of anterior communicating artery (AcoA) score + left posterior communicating artery (PcoA) score + right PcoA score. AcoA score was scored 0 when the AcoA or unilateral A1 segment of the anterior cerebral artery was absent; scored 1 when patients had intact AcoA but A1 diameter was $\leq 50 \%$ of that of the contralateral A1; and scored 2 when the AcoA was intact and both A1 diameters were $>50 \%$ of that of the contralateral A1. For the PcoA score, 0 points were allocated when the PcoA or P1 segment of the posterior cerebral artery was absent; 1 point when the diameter of PcoA was $\leq 50 \%$ of that of the ipsilateral P1; and 2 points when the diameter of PcoA was $>50 \%$ of that of the ipsilateral P1 [17]. Figure 1 shows the examples of this $\mathrm{CoW}$ score. A poor CoW was defined as a CoW score of 0-2 per a prior study [17]. The complete fetal variant of the PcoA (CFPcoA) was defined when patients had visible PcoA but without P1 [18].

\section{MRI-Based CSVD Scores}

MRIs were performed on a 3.0 T MRI machine. All included subject included subjects had axial T1-, T2-weighted images, susceptibility-weighted imaging (SWI), and fluidattenuated inversion recovery. The details for MRI parameters were described in our previous study [19]. The median time from symptom onset to SWI was 5.5 (3.1-15.8) days. MRI CSVD features were rated per the STRIVE consensus criteria [2]. We assessed the presence, number, and severity of lacunes, WMH, EPVS, CMBs, and cSS.

According to a previously described and validated ordinal scores $[8,20,21]$, we rated global CSVD burden (0-6), total HA burden (0-4), and total CAA burden $(0-6)$ for each included patient. In brief, global CSVD burden was rated by allocating 1 point for the presence of lacunes, 1-4 CMBs, EPVS in BG $>20$, and moderate WMH (DWMH + periventricular WMH [PWMH] Fazekas score 3-4), respectively; and 2 points for $\geq 5$ CMBs, and severe WMH (DWMH + PWMH Fazekas score 5-6), respectively [8, 21]. We evaluated the total HA burden by allocating 1 point for the presence of (a) lacunes; (b) $\geq 1$ deep CMB; (c) EPVS in basal ganglia (BG) $>10$; and (d) the presence of WMH (DWMH Fazekas score 2-3 or PWMH Fazekas score 3) $[8,21]$. Finally, the total CAA burden was evaluated by accounting 1 point for (a) 2-4 lobar CMBs; (b) EPVS in centrum semiovale $(\mathrm{CSO})>20$; (c) the presence of WMH; and (d) 
Fig. 1 Examples of the CoW score (range 0-6) according to Kim et al.

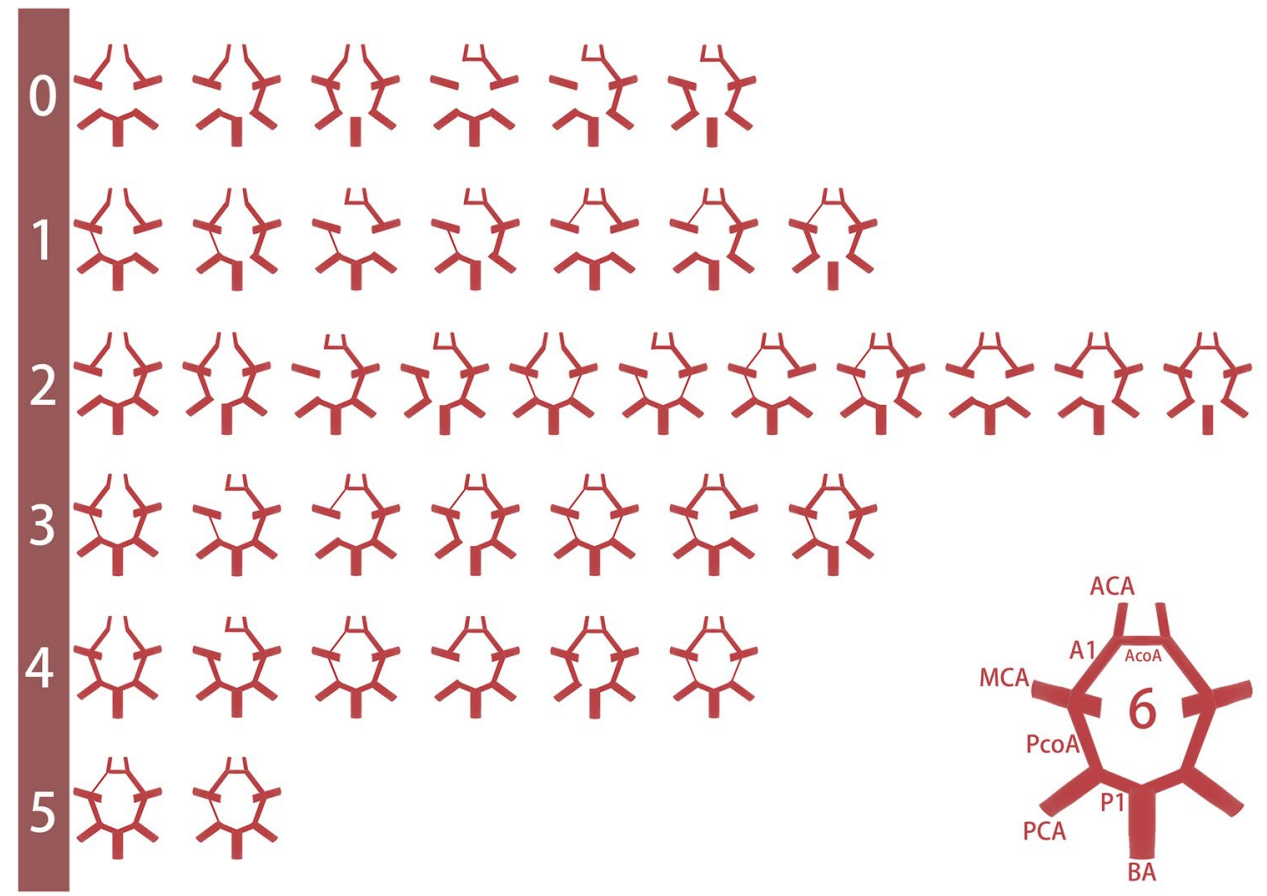

focal cSS (restricted to $1-3$ sulci); and 2 points for (a ) $\geq 5$ lobar CMBs; and (b) disseminated cSS (diffuse to $\geq 4$ sulci) $[8,20]$.

\section{ICH Etiologic Classification}

For all included patients, the ICH etiology was classified as HA-, CAA, mixed-ICH, and undetermined type according to previous studies [13, 14, 22, 23]. HA-ICH was considered when the hemorrhage was located in deep structures (BG, thalamus, and brain stem), with or without deep CMBs, but no lobar CMBs [13, 14]. CAA-ICH was defined when patients had a lobar hemorrhage (cerebellar hemorrhage allowed), aged $\geq 55$ years, with or without lobar CMBs or cSS, but no deep CMBs per the modified Boston criteria for probable, or possible CAA [22]. When patients had hemorrhage/CMBs which were located in both deep and lobar regions, they were classified as having mixed-ICH [23]. For those who had strictly lobar ICH, without deep CMBs, but aged $<55$ years, they were considered as undetermined type. According to the modified Boston criteria [22], lobar ICH/ $\mathrm{CMB}$ which was used to define $\mathrm{ICH}$ etiology included cerebellar location in the present study.

\section{Statistical Methods}

Clinical and neuroimaging characteristics among $\mathrm{HA}-\mathrm{ICH}$, CAA-ICH, and mixed ICH were compared, using ANOVA, Kruskal-Wallis H test, Pearson $\chi 2$, and Fisher exact tests, when appropriate. We used univariable and multivariable logistic regression to look for the associations for $\mathrm{CoW}$ morphology, including ICH etiology, each CSVD features, and three combined CSVD burden. The association between CoW morphology and ICH etiology was adjusted for age, sex, hypertension history, diabetes mellitus (DM) history, smoking, and ICH location (classified as deep, lobar, or cerebellar). Exploratory analyses in subgroups with age and hypertension history were conducted on the basis that both variates were risk factors for both $\mathrm{CoW}$ and ICH [24, 25]. For the association between CoW morphology and CSVD burden, we adjusted for hypertension history, DM history, hyperlipidemia, smoking, and heart disease, as well as age and sex in the multivariable analyses. All tests were performed in IBM SPSS software (version 23). $P$-values $<0.05$ were considered to be statistically significant.

\section{Results}

\section{General Population}

Among the 296 enrolled patients who had brain MRIs and a diagnosis of primary ICH, we excluded 2 patients aged $<18$ years, 70 without CTA/MRA, 8 with prior ischemic stroke, and 1 with the diagnosis of primary intraventricular hemorrhage. Finally, 215 subjects were included into analysis. Overall, there were 94 HA-ICHs, 28 CAA-ICHs, 89 mixedICHs, and 4 undetermined types. The demographic, clinical, and imaging characteristics of the overall population and subgroups by ICH etiology are presented in Table 1. CAA patients were the oldest among HA-, CAA-, and mixed ICH 
Table 1 Clinical and neuroimaging characteristics by ICH etiology

\begin{tabular}{|c|c|c|c|c|}
\hline & Total $(n=215)$ & HA-ICH $(n=94) *$ & CAA-ICH $(n=28)^{*}$ & Mixed ICH $(n=89)^{*}$ \\
\hline Age, mean (SD), y & $60.3(13.4)$ & $55.2(12.5)$ & $71.1(8.6)$ & $63.1(12.5) \dagger$ \\
\hline Male, $n(\%)$ & $157(73.0)$ & $69(73.4)$ & $19(67.9)$ & $66(74.2)$ \\
\hline Hypertension history, $n(\%)$ & $141(65.6)$ & $59(62.8)$ & $15(53.6)$ & $66(74.2)$ \\
\hline DM history, $n(\%)$ & $20(9.3)$ & $7(7.4)$ & $5(17.9)$ & $8(9.0)$ \\
\hline Hyperlipidemia, $n(\%)$ & $8(3.7)$ & $1(1.1)$ & 0 & $6(6.7)$ \\
\hline Alcohol, $n(\%)$ & $45(20.9)$ & $23(24.5)$ & $2(7.1)$ & $18(20.2)$ \\
\hline Smoking, $n(\%)$ & $67(31.2)$ & $28(29.8)$ & $9(32.1)$ & $28(31.5)$ \\
\hline \multicolumn{5}{|l|}{ CSVD burden } \\
\hline Global CSVD burden, median, (IQR) & $3(1-4)$ & $1(0-3)$ & $2(1-4)$ & $4(3-5.5) \dagger$ \\
\hline Total HA burden, median, (IQR) & $2(1-3)$ & $1(1-2)$ & $1(1-2)$ & $3(2-4) \dagger$ \\
\hline Total CAA burden, median, (IQR) & $1(0-3)$ & $1(0-1)$ & $2(1-4)$ & $3(2-4) \dagger$ \\
\hline \multicolumn{5}{|l|}{ CoW parameters } \\
\hline Left A1 diameter, mean (SD), mm & $2.6(0.6)$ & $2.6(0.5)$ & $2.6(0.5)$ & $2.5(0.6)$ \\
\hline Left A1 length, mean (SD), mm & $14.0(2.2)$ & $14.2(2.1)$ & $14.1(2.2)$ & $13.8(2.3)$ \\
\hline Right A1 diameter, mean (SD), mm & $2.4(0.6)$ & $2.4(0.6)$ & $2.4(0.6)$ & $2.5(0.5)$ \\
\hline Right A1 length, mean (SD), mm & $14.5(2.1)$ & $14.3(2.3)$ & $14.3(2.2)$ & $14.8(1.8)$ \\
\hline AcoA diameter, mean (SD), mm & $1.6(0.5)$ & $1.6(0.5)$ & $1.4(0.5)$ & $1.7(0.6)$ \\
\hline Left P1 diameter, mean (SD), mm & $2.5(0.6)$ & $2.4(0.6)$ & $2.5(0.5)$ & $2.5(0.5)$ \\
\hline Left P1 length, mean (SD), mm & $7.8(2.2)$ & $7.5(2.2)$ & $8.1(1.9)$ & $8.1(2.3)$ \\
\hline Left PcoA diameter, mean (SD), mm & $1.7(0.7)$ & $1.7(0.6)$ & $1.6(0.8)$ & $1.7(0.6)$ \\
\hline Left PcoA length, mean (SD), mm & $8.2(2.8)$ & $8.4(2.5)$ & $6.3(3.6)$ & $8.3(2.8)$ \\
\hline Right P1 diameter, mean (SD), mm & $2.5(0.6)$ & $2.4(0.7)$ & $2.4(0.6)$ & $2.5(0.5)$ \\
\hline Right P1 length, mean (SD), mm & $7.2(2.7)$ & $7.4(2.6)$ & $8.3(3.1)$ & $6.6(2.4)$ \\
\hline Right PcoA diameter, mean (SD), mm & $1.9(0.7)$ & $1.9(0.7)$ & $1.7(0.7)$ & $2.1(0.6)$ \\
\hline Right PcoA length, mean (SD), mm & $9.4(2.7)$ & $9.2(2.5)$ & $9.1(2.5)$ & $9.4(3.1)$ \\
\hline CoW score, median, (IQR) & $3(2-4)$ & $3(2-4)$ & $2(2-4)$ & $3(2-4)$ \\
\hline Poor CoW, $n(\%)$ & $102(47.4)$ & $41(43.6)$ & $16(57.1)$ & $43(48.3)$ \\
\hline Incomplete CoW, $n(\%)$ & $161(74.9)$ & $68(72.3)$ & $24(85.7)$ & $67(75.3)$ \\
\hline \multicolumn{5}{|l|}{ CoW category } \\
\hline Complete CoW, $n(\%)$ & $54(25.1)$ & $26(27.7)$ & $4(14.3)$ & $22(24.7)$ \\
\hline Incomplete anterior CoW only, $n(\%)$ & $3(1.4)$ & $2(2.1)$ & 0 & $1(1.1)$ \\
\hline Incomplete posterior CoW only, $n(\%)$ & $122(56.7)$ & $50(53.2)$ & $21(75.0)$ & $50(56.2)$ \\
\hline Incomplete anterior + posterior CoW, $n(\%)$ & $36(16.7)$ & $16(17.0)$ & $3(10.7)$ & $16(18.0)$ \\
\hline CFPcoA, $n(\%)$ & $22(10.2)$ & $7(7.4)$ & $2(7.1)$ & 13 (14.6) \\
\hline
\end{tabular}

*Four patients with lobar ICH, without $\mathrm{CMB}$, and aged < 55 years were classified as undetermined type and were not included into this analysis. $\dagger p<0.001$ among groups. Abbreviation: $I C H$, intracerebral hemorrhage; $H A$, hypertensive arteriopathy; $C A A$, cerebral amyloid angiopathy; $S D$, standard deviation; $D M$, diabetes mellitus; $C S V D$, cerebral small vessel disease; $I Q R$, interquartile range; $C o W$, Circle of Willis; $A c o A$, anterior communicating artery; $P C O A$, posterior communicating artery; $C F P c o A$, complete fetal-variant of PcoA

$(p<0.001)$, and mixed ICH had the highest CSVD burden $(p<0.001$ for each of the three CSVD burden) on univariate analyses.

\section{The Association Between ICH Etiology and CoW Morphology}

Tables 1 and 2 represent the CoW parameters and morphology in the general population and by ICH etiology. Overall, there were no significant differences in the diameter and length of A1, P1, and PcoA, as well as in
CoW score, the presence of poor CoW, incomplete CoW, and CFPcoA. When stratified by age and hypertension, we found that a poor $\mathrm{CoW}$ indicated lower incidence of HA-ICH $(p=0.050)$ in patients aged $\geq 60$ years. While in patients aged $<60$ years, a poor $\mathrm{CoW}$ was associated with a higher incidence of HA-ICH $(p=0.043)$, and lower incidence of mixed ICH ( $p=0.044)$ (Table 2). After correction for age, sex, hypertension history, DM history, smoking, and ICH location, the differences in subgroup analyses of $\geq 60$ years and $<60$ years were not significant (data not shown). Overall, there was a trend towards a 
Table 2 The association of ICH etiology with CoW stratified by age and hypertension*

\begin{tabular}{|c|c|c|c|c|c|c|c|c|c|}
\hline & Non-noor CoW & Poor CoW & $p$-value & Complete CoW & Incomplete $\mathrm{CoW}$ & $p$-value & Non-CFPcoA & CFPcoA & $p$-value \\
\hline \multicolumn{10}{|c|}{$\geq 60$ years $(n=114)$} \\
\hline HA, n (\%) & $18(35.3)$ & $12(19.0)$ & 0.050 & $6(33.3)$ & $24(25.0)$ & 0.560 & $28(28.3)$ & $2(13.3)$ & 0.347 \\
\hline $\mathrm{CAA}, n(\%)$ & $10(19.6)$ & $15(23.8)$ & 0.590 & $2(11.1)$ & $23(24.0)$ & 0.353 & $23(23.2)$ & $2(13.3)$ & 0.516 \\
\hline Mixed, $n(\%)$ & $23(45.1)$ & $36(57.1)$ & 0.201 & $10(55.6)$ & $49(51.0)$ & 0.725 & $48(48.5)$ & $11(73.3)$ & 0.073 \\
\hline \multicolumn{10}{|c|}{$<60$ years $(n=97)$} \\
\hline $\mathrm{HA}, n(\%)$ & $35(58.3)$ & $29(78.4)$ & 0.043 & $20(58.8)$ & $44(69.8)$ & 0.274 & $59(65.6)$ & $5(71.4)$ & 1.000 \\
\hline $\mathrm{CAA}, n(\%)$ & $2(3.3)$ & $1(2.7)$ & 1.000 & $2(5.9)$ & $1(1.6)$ & 0.280 & $3(3.3)$ & 0 & 1.000 \\
\hline Mixed, $n(\%)$ & $23(38.3)$ & 7 (18.9) & 0.044 & $12(35.3)$ & 18 (28.6) & 0.494 & $28(31.1)$ & $2(28.6)$ & 1.000 \\
\hline \multicolumn{10}{|c|}{ With hypertension history $(n=140)$} \\
\hline $\mathrm{HA}, n(\%)$ & $29(45.3)$ & $30(39.5)$ & 0.486 & $13(44.8)$ & $46(41.4)$ & 0.742 & $53(42.4)$ & $6(40.0)$ & 0.859 \\
\hline $\mathrm{CAA}, n(\%)$ & $6(9.4)$ & $9(11.8)$ & 0.638 & $2(6.9)$ & $13(11.7)$ & 0.736 & $14(11.2)$ & $1(6.7)$ & 1.000 \\
\hline Mixed, $n(\%)$ & $29(45.3)$ & $37(48.7)$ & 0.691 & $14(48.3)$ & $52(46.8)$ & 0.891 & $58(46.4)$ & $8(53.3)$ & 0.611 \\
\hline \multicolumn{10}{|c|}{ Without hypertension history $(n=71)$} \\
\hline HA, $n(\%)$ & $24(51.1)$ & $11(45.8)$ & 0.677 & $13(56.5)$ & $22(45.8)$ & 0.399 & $34(53.1)$ & $1(14.3)$ & 0.107 \\
\hline CAA, $n(\%)$ & $6(12.8)$ & $7(29.2)$ & 0.112 & $2(8.7)$ & $11(22.9)$ & 0.199 & $12(18.8)$ & $1(14.3)$ & 1.000 \\
\hline Mixed, $n(\%)$ & $17(36.2)$ & $6(25.0)$ & 0.341 & $8(34.8)$ & $15(31.3)$ & 0.766 & $18(28.1)$ & $5(71.4)$ & $0.032 \dagger$ \\
\hline
\end{tabular}

*Four patients with lobar ICH, without $\mathrm{CMB}$, and aged $<55$ years were classified as undetermined type and were not included into this analysis. $\dagger$ The association between fetal-variant of PcoA and mixed ICH in patients without hypertension was still significant after correction for age, sex, diabetes mellitus history, smoking, and ICH location (OR 17.027, 95\% CI 1.564-185.356, $p=0.020)$. Abbreviation: $H A$, hypertensive arteriopathy; $C A A$, cerebral amyloid angiopathy; $C o W$, Circle of Willis; $P c o A$, posterior communicating artery; $C F P c o A$, complete fetal variant of PcoA

greater incidence of mixed ICH in patients with CFPcoA compared with those without CFPcoA (59.1\% vs $40.2 \%$, $p=0.090)$. In the subgroup of patients without hypertension, the difference reached statistical significance $(71.4 \%$ vs $28.1 \%, p=0.032$ ), even after adjusting for age, sex, DM history, smoking, and ICH location $(O R$ 17.027, $95 \%$ CI
$1.564-185.356, p=0.020)$. Figure 2 shows a representative case without hypertension history had a mixed ICH and fetal variant type of PcoA. There were no significant differences between ICH etiology and both poor CoW and incomplete CoW when stratified by hypertension.
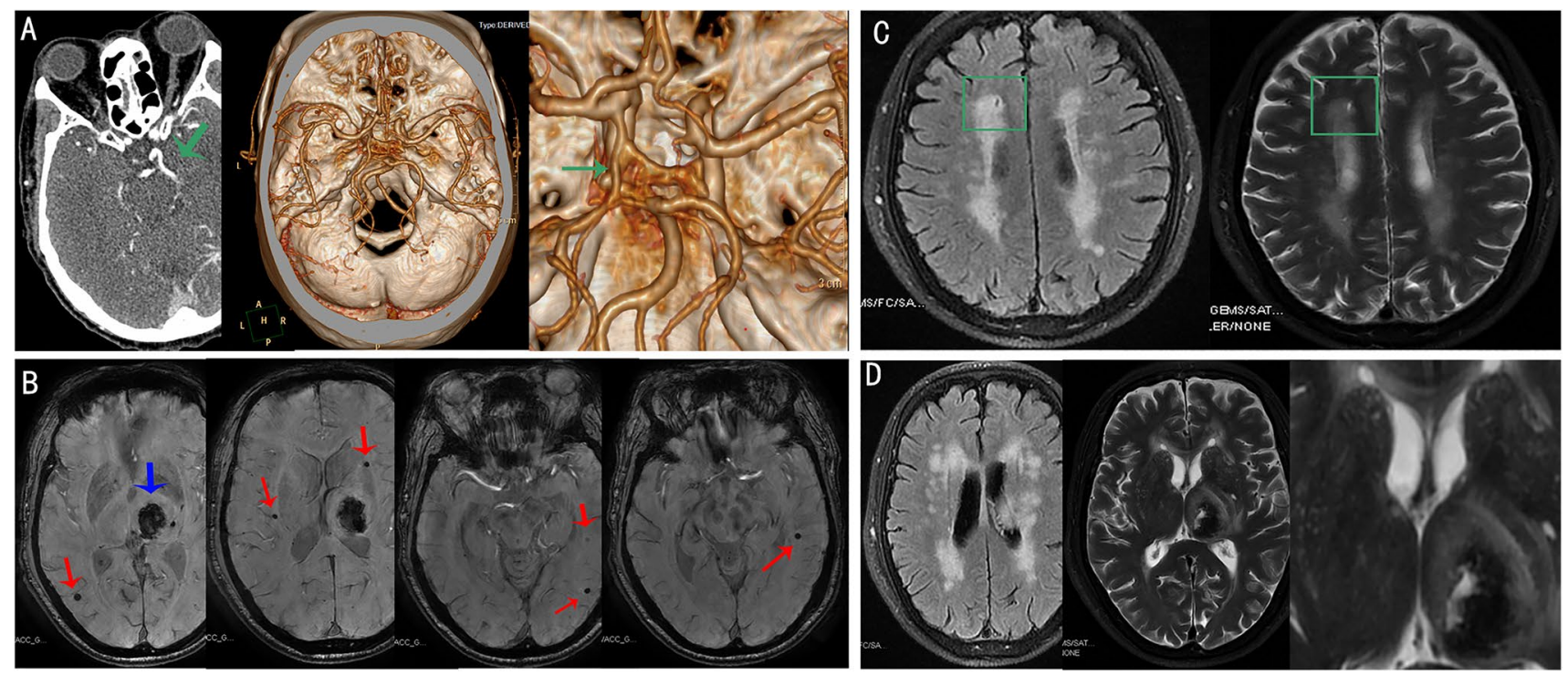

Fig. 2 Representative case of mixed ICH without hypertension history had a fetal-variant of PcoA. A The fetal-variant of CoW (green arrow); $\mathbf{B}$ mixed $\mathrm{CMB} / \mathrm{ICH}$ in SWI sequence (the red arrows show multiple CMBs, and the blue arrow shows an $\mathrm{ICH}$ in deep); $\mathbf{C}$ one lacune in FLAIR and T2-weighted sequences (green box); D severe WMH in both deep and periventricular regions and EPVS $>20$ in BG 


\section{CoW Morphology and CSVD Burden}

Our data suggested that an incomplete CoW was associated with higher incidence of lacunes $(O R 2.020,95 \% C I$ $1.043-3.913, p=0.037)$ and CMBs $\geq 5($ OR 2.426, 95\% CI $1.208-4.872, p=0.013)$, but not with WMH burden, EPVS $>20$ in BG, EVPS > 20 in CSO, and cSS. As expected, the combined CSVD burden was higher in patients with an incomplete CoW (OR 1.198, 95\% CI 1.015-1.413, $p=0.032$ for global CSVD burden; OR $1.304,95 \% C I$ $1.031-1.651, p=0.027$ for total HA burden; OR 1.353, 95\% CI 1.069-1.713, $p=0.012$ for the total CAA burden). Adjustment for hypertension history, DM history, hyperlipidemia, smoking, and heart disease did not alter the direction or strength of the association of incomplete CoW with lacune (adjusted OR 2.114, 95\% CI 1.062-4.207, $p=0.033$ ), CMB $\geq 5$ (adjusted OR 2.437, 95\% CI 1.187-5.002, $p=$ 0.015), global CSVD burden (adjusted $O R$ 1.194, 95\% CI $1.004-1.419, p=0.045$ ), and the total CAA burden (adjusted OR 1.343, 95\% CI 1.056-1.707, $p=0.016$ ). However, after additional adjustment for age and sex, these associations were no longer significant (Table 3).

Poor CoW was not significantly associated with each CSVD feature or each combined CSVD burden either in the general population, or in subgroups stratified with age with a cut off 60 years, or hypertension history (data not shown). Although the presence of CFPcoA did not affect the individual CSVD feature or combined CSVD burden overall (data not shown), fetal variant of PcoA in subgroup of patients without hypertension was significantly associated with high incidence of lacune ( $O R 13.143,95 \% C I$ $1.487-116.152, p=0.021), \mathrm{CMB} \geq 5$ (OR 6.806, 95\% CI $1.211-38.254, p=0.029)$, global CSVD burden (OR 1.814, 95\% CI 1.141-2.884, $p=0.012$ ), total HA burden (OR $2.282,95 \% C I 1.152-4.520, p=0.018)$, and total CAA burden (OR 1.897, 95\% CI 1.183-3.043, $p=0.008)$. Figure 2 also shows the representative case without hypertension history had a fetal variant type of PcoA and high CSVD burden. After correction for DM history, hyperlipidemia, smoking, and heart disease, as well as age and sex, the fetal variant of PcoA still affected the global CSVD burden (adjusted $O R$ 2.025, 95\% CI 1.026-3.997, $p=0.042$ ), total HA burden (adjusted $O R 3.569,95 \% C I 1.106-11.513, p=0.033$ ), and total CAA burden (adjusted OR 2.626, 95\% CI 1.140-6.053, $p=0.023$ ), but not lacune or $\mathrm{CMB} \geq 5$. However, in patients with hypertension, these associations were not significant (data not shown).

\section{Discussion}

The CoW is often investigated in patients with atherosclerotic disease or ischemic stroke [12, 16, 17, 25], but little attention has been given to patients with ICH in the literature. Our

Table 3 The association between CSVD markers and incomplete CoW $(n=215)$

\begin{tabular}{|c|c|c|c|c|c|c|}
\hline & \multicolumn{2}{|l|}{ Univariable analysis } & \multicolumn{2}{|c|}{ Multivariable analysis (model 1) } & \multicolumn{2}{|c|}{ Multivariable analysis (model 2) } \\
\hline & OR $(95 \% C I)$ & $p$-value & Adjusted OR (95\% CI) & $p$-value & Adjusted $O R(95 \% C I)$ & $p$-value \\
\hline Any Lacune & $2.020(1.043-3.913)$ & 0.037 & $2.114(1.062-4.207)$ & 0.033 & $1.802(0.883-3.677)$ & 0.106 \\
\hline $\mathrm{CMB} \geq 1$ & $1.149(0.589-2.241)$ & 0.684 & $1.038(0.511-2.110)$ & 0.917 & $0.903(0.433-1.884)$ & 0.786 \\
\hline $\mathrm{CMBs} \geq 5$ & $2.426(1.208-4.872)$ & 0.013 & $2.437(1.187-5.002)$ & 0.015 & $2.034(0.967-4.276)$ & 0.061 \\
\hline Presence of lobar CMB & $1.375(0.730-2.592)$ & 0.324 & $1.337(0.692-2.582)$ & 0.387 & $1.061(0.531-2.117)$ & 0.867 \\
\hline Lobar CMB $\geq 5$ & $2.003(0.835-4.805)$ & 0.120 & $2.041(0.840-4.959)$ & 0.115 & $1.790(0.717-4.473)$ & 0.212 \\
\hline Total WMH score & $1.136(0.955-1.352)$ & 0.151 & $1.102(0.919-1.321)$ & 0.295 & $0.974(0.798-1.189)$ & 0.797 \\
\hline DWMH Fazekas score & $1.298(0.939-1.793)$ & 0.114 & $1.245(0.889-1.744)$ & 0.202 & $1.034(0.720-1.486)$ & 0.856 \\
\hline PWMH Fazekas score & $1.203(0.867-1.668)$ & 0.269 & $1.126(0.799-1.585)$ & 0.498 & $0.877(0.600-1.283)$ & 0.499 \\
\hline The presence of WMH & $1.631(0.870-3.057)$ & 0.127 & $1.537(0.798-2.962)$ & 0.199 & $1.078(0.532-2.182)$ & 0.836 \\
\hline EPVS in $B G>20$ & $1.421(0.700-2.883)$ & 0.331 & $1.385(0.668-2.872)$ & 0.381 & $0.709(0.301-1.669)$ & 0.431 \\
\hline EPVS in $\mathrm{CSO}>20$ & $1.761(0.917-3.383)$ & 0.089 & $1.668(0.856-3.253)$ & 0.133 & $1.340(0.668-2.688)$ & 0.410 \\
\hline cSS presence & $1.731(0.717-4.179)$ & 0.222 & $1.865(0.754-4.615)$ & 0.177 & $1.187(0.452-3.118)$ & 0.727 \\
\hline Disseminated cSS & $1.369(0.371-5.046)$ & 0.637 & $1.531(0.401-5.847)$ & 0.533 & $0.919(0.226-3.743)$ & 0.906 \\
\hline Global CSVD burden & $1.198(1.015-1.413)$ & 0.032 & $1.194(1.004-1.419)$ & 0.045 & $1.078(0.892-1.302)$ & 0.439 \\
\hline Total HA burden & $1.304(1.031-1.651)$ & 0.027 & $1.282(0.997-1.648)$ & 0.053 & $1.134(0.867-1.483)$ & 0.359 \\
\hline Total CAA burden & $1.353(1.069-1.713)$ & 0.012 & $1.343(1.056-1.707)$ & 0.016 & $1.193(0.925-1.538)$ & 0.173 \\
\hline
\end{tabular}

Model 1: adjusted for hypertension history, diabetes mellitus history, hyperlipidemia, smoking, and heart disease. Model 2: adjusted for factors in model $1+$ age and sex. Abbreviation: $C S V D$, cerebral small vessel disease; $\underline{C o W}$, Circle of Willis; $O R$, odds ratio; $C I$, confidence interval; $C M B$, cerebral microbleed; $W M H$, white matter hyperintensities; $D W M H$, deep WMH; $P W M H$, periventricular WMH; $B G$, basal ganglia; $C S O$, centrum semiovale; $c S S$, cortical superficial siderosis; $H A$, hypertensive arteriopathy; $C A A$, cerebral amyloid angiopathy 
present study in consecutive ICH patients finds that in patients aged $<60$ years, poor $\mathrm{CoW}$ was associated with a higher incidence of HA-ICH, and a lower incidence of mixed ICH, although the significant associations were not independent. It is well-known that patients with HA-ICH were often younger than CAA-ICH patients [14]. And data from our present study and previous reports $[23,26]$ indicated that patients with mixed-ICH were older than those with HA-ICH, but younger than patients with CAA-ICH. Therefore, the higher incidence of $\mathrm{HA}-\mathrm{ICH}$ in patients with poor $\mathrm{CoW}$ in those with younger age is expected. The impact of poor $\mathrm{CoW}$ in $\mathrm{ICH}$ etiology might be indirect. Vrselja et al. [27] have pointed out that $\mathrm{CoW}$ and its communicating arteries protect cranial microvasculature and blood-brain barrier by functioning as a pressure absorber mechanism (i.e., receive great blood pressure burden) in high blood pressure condition. In addition, penetrating arterioles in deep brain regions, which have stiff and brittle structure due to the degeneration of focal smooth muscle cell and collagen deposition in the vessel walls, are prone to burst because of their proximity to the CoW [28]. Therefore, the poor CoW, which might partly lose the ability to absorb great pressure, could lead to deteriorate the stiff and brittle penetrating arterioles which lie downstream of the $\mathrm{CoW}$, and then bring into an ICH prone situation. However, this assumption needs to be verified in future studies.

The presence of CFPcoA was significantly associated with greater incidence of mixed ICH in patients without hypertension, independent of age, sex, DM history, smoking, and ICH location. Interestingly, fetal variant of PcoA also affected CSVD burden, with higher presence of CFPcoA, more severe global CSVD burden, total HA burden, and total CAA burden, even after adjusting for age, sex, and vascular risk factors. As shown in Table 1 in our present study and previous studies [23, 26], mixed ICH had the highest CSVD burden except for cSS which is a MRI marker for CAA by the modified Boston criteria [22]. Therefore, it is reasonable that patients with CFPcoA would more likely to have mixed ICH when compared with HA- or CAA-ICH. Previous studies have shown that in cases with fetal variant of PcoA, vascular insufficiency is more prone to be developed than non-fetal variant of PcoA because of the nature of this anatomy variants [29]. In addition, an ischemia status caused by carotid stenosis $\geq 25 \%$ is associated with deep and mixed CMB [30], where patients with mixed CMB were classified as having mixed ICH. Taken together, fetal-variant PcoA indicates a high likelihood of small vessel disease in both deep and lobar regions, which could be caused by severe HA, or the mixture of HA and CAA [23, 26, 31]. Our results indicate that in the absence of a history of hypertension, fetal-variant PcoA might be another important factor in triggering severe CSVD.

Another important finding is the association of incomplete of CoW with CSVD burden in ICH patients. A previous study showed that individuals with incompleteness of the anterior CoW defined as absent A1 segment correlated with a higher frequency of lacunes in healthy individuals [11]. In the present study, we found a higher frequency of lacunes either in patients with incomplete CoW or in patients with incomplete posterior CoW (OR 2.257, 95\% CI $1.170-4.354, p=0.015$ ), but not in those with incomplete anterior CoW. The different result about incomplete anterior CoW and lacune could be explained by the different study population and that the frequency of absent A1 was only $1.9 \%(4 / 215)$. Also, we found that the presence of CFPcoA correlates with a higher presence of lacune, especially in subgroup of patients without hypertension, after correction for age, sex, and vascular risk factors. Our present study expands our understanding of CoW morphology and lacunes in addition to previously published study by Miyazawa et al. [11]. As for WMH, Ryan et al. [9] found a greater WMH burden in subjects with incomplete CoW; however, we did not found any difference between patients with and without incomplete CoW either in total WMH score or in DWMH, while the latter is in accordance with van der Grond et al.'s finding [12]. The main difference between our study and the study by Ryan et al. [9] is that Ryan et al. evaluated WMH in older patients (aged greater than 50 years), and these subjects might have a higher white matter lesion load than ours. Furthermore, the different score systems of WMH could also be an underlying factor that influences the result. In this respect, future studies are needed to investigate the association of CoW morphology with quantitative WMH burden.

Of note, our findings also suggest that most patients with an incomplete CoW or CFPcoA, especially in patients without hypertension history had severe CSVD burden. With regard to the high stroke recurrence and unfavorable functional outcome in ICH patients with severe CSVD burden $[6,8]$, the variant of CoW has significant clinical meaning that it might have an impact on the mechanisms of $\mathrm{ICH}$, while potentially aiding prevention of stroke onset and stroke recurrence.

Our study had several limitations. We included participants who had brain MRIs, and therefore there might be selection bias. However, MRI, particularly SWI or gradient echo T2* sequence, is useful for detecting CMB or cSS, making it a good choice for careful identification of the ICH mechanisms to optimizing management [4]. Furthermore, MRI is more sensitive to small changes of small vessel diseases [3]. In our present study, we assessed not only individual CSVD features, but also the combined CSVD burden using different weightings to overall reflect the severity of CSVD burden. Second, the sample size was relatively small, resulting in the wide $95 \% \mathrm{CI}$ in subgroup analyses in patients with and without hypertension. Studies with larger sample size are necessary to confirm our results and to explore the underlying mechanism. 


\section{Conclusions}

In summary, our study shows that CoW morphology has an important role in $\mathrm{ICH}$, for instance, CFPcoA affects the occurrence of mixed $\mathrm{ICH}$, and both incomplete $\mathrm{CoW}$ and fetal variant of PcoA are associated with high CSVD burden. Our findings are novel, and need to be verified in future independent study. If replicated, our results help to improve our understanding of the mechanisms of primary $\mathrm{ICH}$ and have important implications for the risk assessment for mixed ICH.

Funding The study was supported by the 1.3.5 Project for Disciplines of Excellence-Clinical Research Incubation Project of West China Hospital (2018HXFH041), the National Natural Science Foundation of China (82001250), China Postdoctoral Science Foundation (2020M683322, 2021T140488), and the 1.3.5 Project for Disciplines of Excellence, West China Hospital, Sichuan University (ZYGD18009).

Data Availability Data will be made available on reasonable request.

\section{Declarations}

Ethics Approval This study was approved by Ethics Committee on Biomedical Research, West China Hospital of Sichuan University.

Consent for Publication Not applicable.

Conflicts of Interest The authors declare no competing interests.

Open Access This article is licensed under a Creative Commons Attribution 4.0 International License, which permits use, sharing, adaptation, distribution and reproduction in any medium or format, as long as you give appropriate credit to the original author(s) and the source, provide a link to the Creative Commons licence, and indicate if changes were made. The images or other third party material in this article are included in the article's Creative Commons licence, unless indicated otherwise in a credit line to the material. If material is not included in the article's Creative Commons licence and your intended use is not permitted by statutory regulation or exceeds the permitted use, you will need to obtain permission directly from the copyright holder. To view a copy of this licence, visit http://creativecommons.org/licenses/by/4.0/.

\section{References}

1. Qureshi AI, Tuhrim S, Broderick JP, Batjer HH, Hondo H, Hanley DF. Spontaneous intracerebral hemorrhage. The New England journal of medicine. 2001;344:1450-60.

2. Wardlaw JM, Smith EE, Biessels GJ, Cordonnier C, Fazekas F, Frayne R, et al. Neuroimaging standards for research into small vessel disease and its contribution to ageing and neurodegeneration. The Lancet Neurology. 2013;12:822-38.

3. Wardlaw JM, Smith C, Dichgans M. Small vessel disease: mechanisms and clinical implications. The Lancet Neurology. 2019;18:684-96.
4. Schrag M, Kirshner H. Management of intracerebral hemorrhage: JACC Focus Seminar. Journal of the American College of Cardiology. 2020;75:1819-31.

5. Akoudad S, Portegies ML, Koudstaal PJ, Hofman A, van der Lugt A, Ikram MA, et al. Cerebral microbleeds are associated with an increased risk of stroke: the Rotterdam study. Circulation. 2015;132:509-16.

6. Xu M, Li B, Zhong D, Cheng Y, Wu Q, Zhang S, et al. Cerebral small vessel disease load predicts functional outcome and stroke recurrence after intracerebral hemorrhage: a median follow-up of 5 years. Frontiers in aging neuroscience. 2021;13:628271.

7. Charidimou A, Imaizumi T, Moulin S, Biffi A, Samarasekera N, Yakushiji Y, et al. Brain hemorrhage recurrence, small vessel disease type, and cerebral microbleeds: a meta-analysis. Neurology. 2017;89:820-9.

8. Pasi M, Sugita L, Xiong L, Charidimou A, Boulouis G, Pongpitakmetha T, et al. Association of cerebral small vessel disease and cognitive decline after intracerebral hemorrhage. Neurology. 2021;96:e182-e92.

9. Ryan DJ, Byrne S, Dunne R, Harmon M, Harbison J. White matter disease and an incomplete circle of Willis. International journal of stroke : official journal of the International Stroke Society. 2015; 10:547-52.

10. Warnert EA, Rodrigues JC, Burchell AE, Neumann S, Ratcliffe LE, Manghat NE, et al. Is high blood pressure self-protection for the brain? Circulation research. 2016;119:e140-e51.

11. Miyazawa N, Shinohara T, Yamagata Z. Association of incompleteness of the anterior part of the circle of Willis with the occurrence of lacunes in the basal ganglia. European journal of neurology. 2011;18:1358-60.

12. van der Grond J, van Raamt AF, van der Graaf Y, Mali WP, Bisschops RH. A fetal circle of Willis is associated with a decreased deep white matter lesion load. Neurology. 2004;63:1452-6.

13. Pasi M, Boulouis G, Fotiadis P, Auriel E, Charidimou A, Haley K, et al. Distribution of lacunes in cerebral amyloid angiopathy and hypertensive small vessel disease. Neurology. 2017;88:2162-8.

14. Charidimou A, Boulouis G, Haley K, Auriel E, van Etten ES, Fotiadis $\mathrm{P}$, et al. White matter hyperintensity patterns in cerebral amyloid angiopathy and hypertensive arteriopathy. Neurology. 2016;86:505-11.

15. Krabbe-Hartkamp MJ, van der Grond J, de Leeuw FE, de Groot JC, Algra A, Hillen B, et al. Circle of Willis: morphologic variation on three-dimensional time-of-flight MR angiograms. Radiology. 1998;207:103-11.

16. Millesi K, Mutzenbach JS, Killer-Oberpfalzer M, Hecker C, Machegger L, Bubel N, et al. Influence of the circle of Willis on leptomeningeal collateral flow in anterior circulation occlusive stroke: Friend or foe? Journal of the neurological sciences. 2019;396:69-75.

17. Kim KM, Kang HS, Lee WJ, Cho YD, Kim JE, Han MH. Clinical significance of the circle of Willis in intracranial atherosclerotic stenosis. Journal of neurointerventional surgery. 2016;8:251-5.

18. Hong JM, Choi JY, Lee JH, Yong SW, Bang OY, Joo IS, et al. Impact of posterior communicating artery on basilar artery stenoocclusive disease. Journal of neurology, neurosurgery, and psychiatry. 2009;80:1390-3.

19. Xu M, Cheng Y, Song Q, Yuan R, Zhang S, Hao Z, et al. Total burden of cerebral small vessel disease in recurrent $\mathrm{ICH}$ versus first-ever ICH. Aging and disease. 2019;10:570-7.

20. Charidimou A, Martinez-Ramirez S, Reijmer YD, Oliveira-Filho J, Lauer A, Roongpiboonsopit D, et al. Total magnetic resonance imaging burden of small vessel disease in cerebral amyloid angiopathy: an imaging-pathologic study of concept validation. JAMA neurology. 2016;73:994-1001. 
21. Doubal FN, MacLullich AM, Ferguson KJ, Dennis MS, Wardlaw JM. Enlarged perivascular spaces on MRI are a feature of cerebral small vessel disease. Stroke. 2010;41:450-4.

22. Linn J, Halpin A, Demaerel P, Ruhland J, Giese AD, Dichgans M, et al. Prevalence of superficial siderosis in patients with cerebral amyloid angiopathy. Neurology. 2010;74:1346-50.

23. Tsai HH, Pasi M, Tsai LK, Chen YF, Lee BC, Tang SC, et al. Microangiopathy underlying mixed-location intracerebral hemorrhages/microbleeds: a PiB-PET study. Neurology. 2019;92:e774-e81.

24. Gross BA, Jankowitz BT, Friedlander RM. Cerebral intraparenchymal hemorrhage: a review. Jama. 2019;321:1295-303.

25. Shuaib A, Butcher K, Mohammad AA, Saqqur M, Liebeskind DS. Collateral blood vessels in acute ischaemic stroke: a potential therapeutic target. The Lancet Neurology. 2011;10:909-21.

26. Pasi M, Charidimou A, Boulouis G, Auriel E, Ayres A, Schwab $\mathrm{KM}$, et al. Mixed-location cerebral hemorrhage/microbleeds: underlying microangiopathy and recurrence risk. Neurology. 2018;90:e119-e26.

27. Vrselja Z, Brkic H, Mrdenovic S, Radic R, Curic G. Function of circle of Willis. Journal of cerebral blood flow and metabolism
: official journal of the International Society of Cerebral Blood Flow and Metabolism. 2014;34:578-84.

28. Ratelade J, Klug NR, Lombardi D, Angelim M, Dabertrand F, Domenga-Denier V, et al. Reducing hypermuscularization of the transitional segment between arterioles and capillaries protects against spontaneous intracerebral hemorrhage. Circulation. 2020;141:2078-94.

29. van Raamt AF, Mali WP, van Laar PJ, van der Graaf Y. The fetal variant of the circle of Willis and its influence on the cerebral collateral circulation. Cerebrovascular diseases (Basel, Switzerland). 2006;22:217-24.

30. Romero JR, Preis SR, Beiser A, DeCarli C, D'Agostino RB, Wolf PA, et al. Carotid atherosclerosis and cerebral microbleeds: the Framingham heart study. Journal of the American Heart Association. 2016;5:e002377.

31. Blanc C, Viguier A, Calviere L, Planton M, Albucher JF, Rousseau $\mathrm{V}$, et al. Underlying small vessel disease associated with mixed cerebral microbleeds. Frontiers in neurology. 2019;10:1126.

Publisher's Note Springer Nature remains neutral with regard to jurisdictional claims in published maps and institutional affiliations. 